\title{
Análisis de la longitud de las rectas y su influencia en la consistencia del diseño geométrico de carreteras convencionales
}

\author{
Alfredo García \\ Catedrático, Grupo de Investigación en Ingeniería de Carreteras (GIIC), Universitat \\ Politècnica de València (UPV), España \\ Francisco J. Camacho-Torregrosa \\ Ayudante Doctor, GIIC, UPV, España \\ Jaime S. Díaz García \\ Ingeniero de Caminos, C. y P., España
}

\section{RESUMEN}

Los trazados que presentan rectas de elevada longitud fomentan que los conductores circulen a mayores velocidades, pudiendo alcanzar sus velocidades deseadas en la parte central de las mismas. Como consecuencia de ello, son grandes las reducciones de velocidad para afrontar las curvas posteriores. En cambio, con rectas cortas, las velocidades que se pueden desarrollar en las dos curvas de los extremos están condicionadas mutuamente para que no haya una reducción excesiva de la velocidad. Las reducciones excesivas de velocidad comprometen la adecuada consistencia de una carretera por lo que están correlacionadas con una mayor siniestralidad.

La mayoría de modelos de velocidad de operación existentes han sido calibrados a partir de mediciones puntuales de velocidad. Para estimar los perfiles de velocidad de operación, se hace necesario incorporar unas reglas de construcción. La gran mayoría de ellas considera que los cambios de velocidad se producen solo a lo largo de la recta. En la investigación que se presenta, se ha analizado el comportamiento operacional de un gran número de conductores en las rectas, encontrando importantes diferencias respecto del comportamiento anterior. Analizando la evolución de la velocidad de operación observada en 70 secciones curva-recta-curva de carreteras convencionales se ha encontrado que la variación de velocidad se produce en una longitud superior a la propia recta; esa nueva longitud efectiva está conformada por la longitud de la recta más un cierto desarrollo de las curvas adyacentes. Los resultados apuntan a que la longitud empleada para variar la velocidad antes y después de las rectas es de unos $145 \mathrm{~m}$, independientemente de la configuración geométrica.

Adicionalmente, se han comparado los resultados del análisis geométrico y operacional de las secciones con los criterios correspondientes de la nueva Instrucción de Trazado de Carreteras, encontrando disparidades manifiestas y planteándose mejoras a la misma, siendo el punto de mayor discrepancia las reducidas longitudes que esta emplea para considerar algunas rectas como no independientes. Se han encontrado tres niveles de independencia de las rectas a diferencia de los dos niveles tradicionalmente empleados a nivel internacional. 


\section{INTRODUCCIÓN}

En carreteras convencionales, las rectas de cierto desarrollo son necesarias para poder facilitar el adelantamiento y así aliviar las demoras de los vehículos más rápidos, mejorando así el nivel de servicio. Como contrapartida, rectas largas propician mayores velocidades. En los casos de longitudes muy elevadas, los conductores pueden alcanzar su velocidad deseada, que mantendrán constante hasta aproximarse a la curva posterior. Este comportamiento es especialmente relevante cuando las reducciones de velocidad previas a encarar la siguiente curva son de gran magnitud. Este tipo de rectas largas se denominan rectas independientes (Lamm et al., 2007), al no estar condicionadas por las curvas.

En cambio, cuando la recta es corta, las velocidades que se pueden desarrollar están muy relacionadas con las de las curvas debido al poco desarrollo existente para variar la velocidad. En casos extremos, puede haber incluso transición directa de velocidad entre ellas. Este tipo de rectas se denominan no independientes (Lamm et al., 2007) o dependientes.

La velocidad de operación es entendida como la velocidad que desarrollan los usuarios de la carretera bajo condiciones de flujo libre. Esta velocidad de operación para cada elemento del trazado está reconocida que se corresponde con el percentil 85 de las velocidades en flujo libre de vehículos ligeros.

Se han desarrollado diversos modelos para la estimación de la velocidad de operación en rectas, si bien su estimación no es sencilla, al no ejercer estas un control geométrico sobre los conductores. Así, podemos encontrar como modelos más simples una velocidad única para todas las rectas independientes: Ottesen y Krammes (2000) propusieron 97,9 km/h; Fitzpatrick y Collins (2000) adoptaron $100 \mathrm{~km} / \mathrm{h}$; Easa (2003) la seleccionaba en el intervalo $(94 ; 104) \mathrm{km} / \mathrm{h}$, estimándose aproximadamente en $100 \mathrm{~km} / \mathrm{h}$ en vías convencionales con velocidad límite de $90 \mathrm{~km} / \mathrm{h}$. Otros modelos más avanzados han tenido en cuenta algunas variables: Fitzpatrick et al. (2000) estimaban la velocidad media de las rectas de longitud superior a 200 m en función de diferentes índices de trazado; Polus et al. (2000) se basaban en los radios de las curvas de los extremos y la longitud de la recta; Pérez et al. (2010) propusieron dos modelos que dependían de la longitud de la recta y las características geométricas y operacionales de la curva anterior.

Las reducciones excesivas de velocidad comprometen la adecuada consistencia de una carretera por lo que están correlacionadas con una mayor siniestralidad. Puede definirse la consistencia del diseño geométrico como el grado de adecuación entre el comportamiento de la carretera y las expectativas de los conductores (Nicholson, 1998). Un diseño inconsistente da como resultado sorpresas en los conductores $\mathrm{y}$, por tanto, mayor probabilidad de existir un comportamiento anómalo que derive en un accidente. La mayoría de las investigaciones relacionadas con la consistencia y los modelos más empleados se 
centran en la velocidad de operación y sus variaciones (Hassan, 2004), por ser relativamente sencilla su estimación pero a la vez ofrecer buenos resultados. Esta velocidad se emplea para evaluar la consistencia básicamente de dos formas: examinando su variación a lo largo de la vía (buscando deceleraciones bruscas o altas variaciones), o comparándola con la velocidad de diseño.

En los análisis de consistencia, las transiciones recta-curva son consideradas como las ubicaciones más críticas, ya que se estima que más del $50 \%$ de las muertes en carreteras interurbanas se producen en secciones curvas (Lamm et al., 1992). La reducción de velocidad de operación entre dos elementos consecutivos $(\Delta V 85)$ es una forma indirecta de cuantificar la sorpresa experimentada por los conductores, y se ha revelado como un indicador de consistencia muy sencillo y utilizado. Este es el modelo propuesto por Lamm et al. (1999) y denominado Criterio II, que establece dos umbrales de 10 y $20 \mathrm{~km} / \mathrm{h}$, de la reducción de velocidad de operación entre la recta y la curva siguiente, para distinguir una consistencia buena, aceptable o pobre. Otro modelo más reciente ha sido propuesto por García et al. (2013) para evaluar la consistencia en las curvas empleando el Índice de Consistencia Inercial (ICI). Este nuevo índice consiste en el cálculo en el punto de inicio de la curva de la diferencia entre la velocidad de operación media del kilómetro anterior (velocidad de operación inercial) y la velocidad de operación en ese punto (velocidad de operación de la curva). Posteriormente, se ha fijado la longitud óptima del segmento anterior para promediar la velocidad de operación en 750 m (García et al., 2015).

\section{OBJETIVOS E HIPÓTESIS}

La mayoría de modelos de velocidad de operación consideran que los cambios de velocidad se producen exclusivamente a lo largo del desarrollo de la recta, manteniéndose en los tramos circulares una velocidad constante. En esta investigación se ha introducido una nueva hipótesis, contraria al conocimiento y la práctica previa, según la cual, los conductores inician su aceleración antes de que concluya la primera curva y completan su deceleración ya dentro de la segunda curva. Por tanto, se plantea que, a efectos operacionales, hay una longitud de recta efectiva mayor que la longitud geométrica.

El objetivo principal de la investigación es la mejora del conocimiento sobre cómo influye la longitud de las rectas en la consistencia del diseño geométrico. Para ello se han analizado datos existentes, los cuales fueron obtenidos mediante la observación continua de la evolución de una muestra de vehículos recorriendo diversos tramos de carretera convencional.

Se han planteado las siguientes hipótesis adicionales a verificar mediante el desarrollo experimental:

- Una mayor longitud de recta induce a los conductores a aproximarse más a su velocidad deseada. 
- A menor velocidad de diseño del tramo, menor es la longitud mínima de la recta para ser considera independiente.

- Existe condicionamiento operacional entre curvas consecutivas para rectas con desarrollo por debajo de cierta longitud.

\section{METODOLOGÍA}

La base experimental de esta investigación fue la observación continua de la evolución de muestras de vehículos ligeros en nueve segmentos de carretera convencional. Para ello se emplearon dispositivos GPS de rastreo pasivo de $1 \mathrm{~Hz}$, que se entregaban a los conductores previa aceptación de colaboración. Para esto se siguió la metodología propuesta por Pérez et al. (2013). Así, se disponía de muestras de perfiles individuales de velocidad en varios segmentos de carretera convencional. Se considera segmento aquel tramo de carretera de características geométricas y operacionales aproximadamente constantes, sin intersecciones intermedias y de longitud suficiente para que los conductores desarrollen libremente sus velocidades. En la Tabla 1 se observan la localización y características básicas de los nueve segmentos, todos ellos situados en la provincia de Valencia.

\begin{tabular}{|l|c|c|}
\hline Segmento & $\begin{array}{c}\text { Longitud } \\
(\mathrm{km})\end{array}$ & $\begin{array}{c}\text { IMD } \\
(\mathrm{veh} / \mathrm{día})\end{array}$ \\
\hline CV-35 [Tuéjar-Titaguas] & 13,4 & 860 \\
\hline CV-35 [Calles - Losa del Obispo] & 8,2 & 2257 \\
\hline CV-33 [Urb. Brugar-Pedravilla] & 5,1 & 2419 \\
\hline CV-50 [Cheste-Villamarchante] & 5,7 & 4852 \\
\hline CV-372 [La Pobla-Ribaroja] & 4,5 & 2023 \\
\hline CV-305 [CV-315-CV-310] & 4,4 & 1457 \\
\hline CV-370 [Villamarchante-Pedralba] & 8,3 & 2523 \\
\hline CV-401 [Alfafar-El Saler] & 7,3 & 5292 \\
\hline
\end{tabular}

Tabla 1 - Características de los segmentos de carretera convencional observados

De cada segmento se restituyó la geometría mediante software desarrollado a tal efecto (Camacho-Torregrosa et al., 2015). A partir de la geometría en planta, se seleccionó un total de 70 secuencias curva-recta-curva de diversas características. En estas secciones la longitud de recta varía entre $31 \mathrm{~m}$ y $1446 \mathrm{~m}$, siendo el promedio de $409 \mathrm{~m}$. Las curvas adyacentes presentan radios entre $66 \mathrm{~m}$ y $963 \mathrm{~m}$, con un radio medio de $340 \mathrm{~m}$.

Para la realización del estudio de la velocidad desarrollada en tramos rectos, es necesario utilizar aquellas rectas en las que se llega a alcanzar una velocidad aproximadamente constante, fuera de las zonas de aceleración y deceleración. Por ello, los tramos rectos de carretera utilizados en este estudio se han identificado a partir de los perfiles continuos de velocidad, seleccionando aquellos en los que la velocidad se mantiene constante durante una 
cierta parte de ellos, diferenciándose claramente de los tramos de aceleración y deceleración.

De cada sección de análisis y de su correspondiente perfil de velocidad de operación, se han determinado una serie de variables a partir de las cuales se han realizado todos los cálculos necesarios para el posterior análisis. Estas variables se han agrupado de la siguiente forma:

- Características del segmento (s):

○ Ls: longitud (m)

- Rms: radio mínimo: menor radio de las curvas del segmento (m)

- CCRs: índice de cambio de curvatura (gon $/ \mathrm{km})$

○ V85ms: velocidad de operación media $(\mathrm{km} / \mathrm{h})$

○ V85mxs: velocidad de operación máxima $(\mathrm{km} / \mathrm{h})$

- V85mis: velocidad de operación mínima ( intersecciones que la reduzcan $)(\mathrm{km} / \mathrm{h})$

○ Vds: velocidad de diseño $(\mathrm{km} / \mathrm{h})$

- Características de la sección de análisis (j):

- Geometría:

- Curva 1:

- $\mathrm{R} 1 \mathrm{js}$ : radio (m)

- A11js: parámetro clotoide entrada (m)

- A12js: parámetro clotoide salida (m)

- W1js: ángulo de deflexión total (gon)

- L1js: longitud total (m)

- Lc11js: longitud clotoide entrada (m)

- Lc12js: longitud clotoide salida (m)

- Lr1js: longitud arco círculo (m)

- PKi1js: PK inicial

- PKf1js: PK final

- CCR1js: índice de cambio de curvatura (gon $/ \mathrm{km})$

- Recta:

- Ltjs: longitud (m)

- PKitjs: PK inicial (PKitjs = PKf1js)

- PKftjs: PK final (PKftjs=PKi2js)

- CCRjs: índice de cambio de curvatura de la sección completa (incluyendo ambas curvas) (gon/km)

- Curva 2:

- R2js: radio (m)

- A21js: parámetro clotoide entrada (m)

- A22js: parámetro clotoide salida (m)

- W2js: ángulo de deflexión total (gon) 
- L2js: longitud total (m)

- Lc21js: longitud clotoide entrada (m)

- Lc22js: longitud clotoide salida (m)

- Lr2js: longitud arco círculo (m)

- PKi2js: PK inicial

- PKf2js: PK final

- CCR2js: índice de cambio de curvatura (gon/km)

○ Operación:

- Curva 1:

- V851js: velocidad mínima de operación $(\mathrm{km} / \mathrm{h})$

- PK851js: PK final de velocidad mínima de operación

- Recta:

- V852js: velocidad máxima de operación inicial $(\mathrm{km} / \mathrm{h})$

- PK852js: PK inicial de velocidad máxima de operación

- V853js: velocidad máxima de operación final (no tiene por qué ser igual a V852js) $(\mathrm{km} / \mathrm{h})$

- PK853js: PK final de velocidad máxima de operación

- Curva 2:

- V854js: velocidad mínima de operación $(\mathrm{km} / \mathrm{h})$

- PK854js: PK inicial de velocidad mínima de operación

- Características globales:

- Adelanto del inicio de aceleración: Lt1js=PKitjs-PK851js (m)

- Retraso del final de deceleración: Lt2js=PK854js-PKftjs (m)

○ Longitud efectiva de recta: Ltejs $=$ Ltjs + Lt1js+Lt2js (m)

- Grado de velocidad de operación deseada: GV85djs= V85mxs- $\max (V 852 j s$; V853js) $(\mathrm{km} / \mathrm{h})$

○ Incremento de velocidad de operación en aceleración: $\Delta$ V85ajs= V852jsV851js $(\mathrm{km} / \mathrm{h})$

- Tasa media de aceleración de operación: a85js (m/s2)

○ Disminución de velocidad de operación en deceleración: $\Delta$ Vdjs= V854jsV853js $(\mathrm{km} / \mathrm{h})$

- Tasa media de deceleración de operación: d85js $(\mathrm{m} / \mathrm{s} 2)$

En la Figura 1 se observa cómo se determinan las variables principales asociadas a una sección de análisis, con base en el diagrama de curvaturas y el perfil de velocidad de operación. Básicamente, se trata de localizar en el perfil de velocidad de operación los cuatro puntos característicos de variación de la velocidad (1, 2, 3 y 4). 


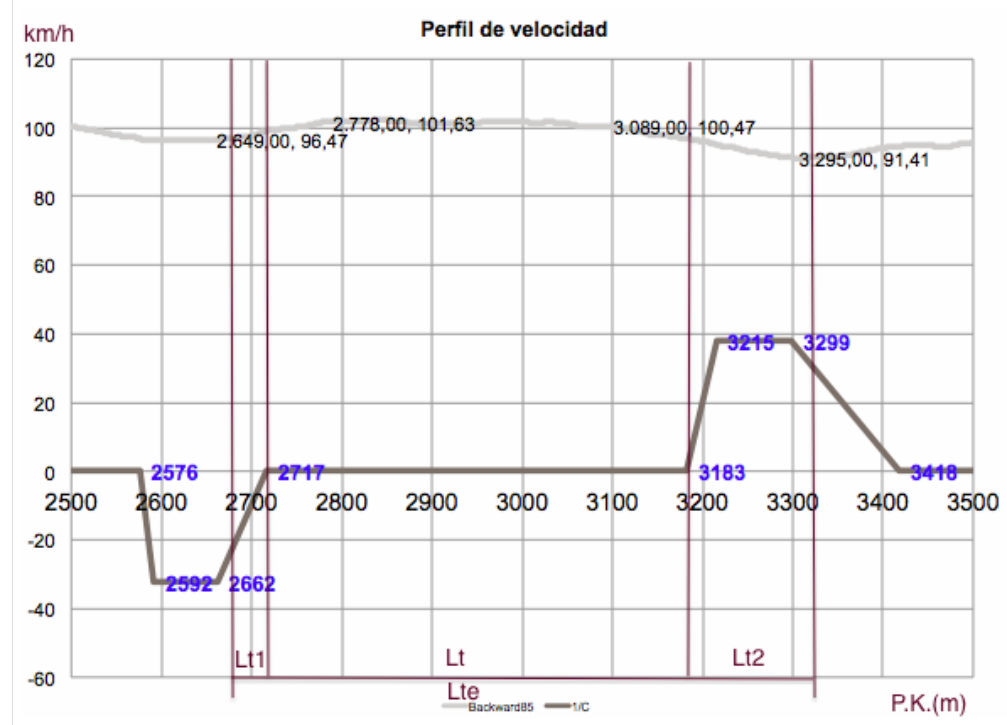

Figura 1 - Esquema de determinación de las variables principales en un perfil de velocidad de operación de una sección de análisis

\section{ANÁLISIS}

Las primeras variables importantes son las longitudes de las curvas que son utilizadas para llevar a cabo variación de la velocidad. La longitud de curva inicial empleada para acelerar (Lt1) presenta un valor medio de 81,0 m, con un máximo de $336 \mathrm{~m}$. Solo hay una sección de análisis donde este valor es negativo, es decir, que no se acelera dentro de la curva. La longitud de curva final empleada para decelerar (Lt2) arroja un valor medio de 82,2 m, con un máximo de $340 \mathrm{~m}$. En este caso hay dos secciones donde la deceleración concluye antes de terminar la recta. Hay que recalcar que las longitudes anteriores no constituyen el total de longitud empleada para cambiar la velocidad, sino la porción que corresponde a los tramos curvos.

Sumando ambas longitudes a la propia de la recta obtenemos una longitud efectiva (Lte) superior a la longitud geométrica de la recta $(\mathrm{Lt})$. Esta operación se ha realizado para todas las rectas, obteniendo la gráfica de la Figura 2. El ajuste lineal da como resultado una muy alta correlación entre las longitudes, con pendiente próxima a la unidad. Forzando la pendiente de la recta de ajuste a la unidad se obtiene que la longitud efectiva es igual a la longitud geométrica de la recta añadiéndole $142,59 \mathrm{~m}$, independientemente de dicha longitud geométrica. 


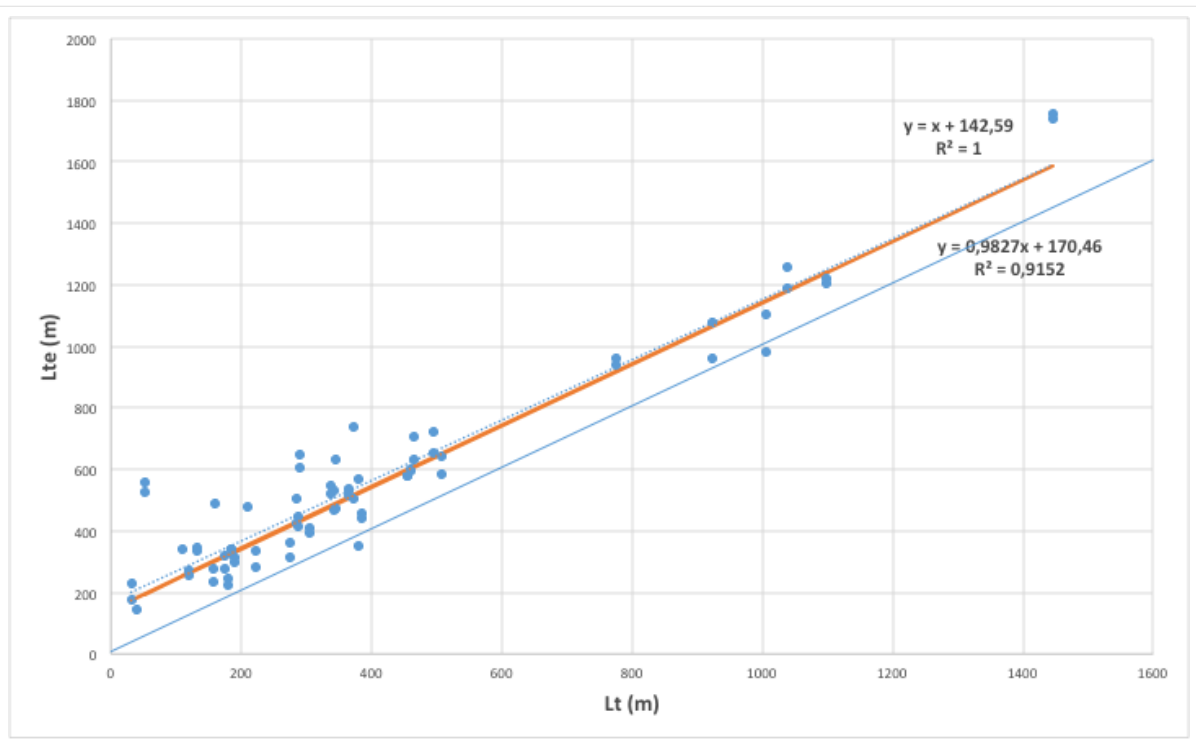

Figura 2 - Relación entre la longitud geométrica y efectiva de las rectas

Así se ha verificado la primera hipótesis planteada, es decir, las variaciones de velocidad no se producen solo a lo largo de la recta intermedia. Además, el modelo resultante para la longitud de la recta efectiva, desde el punto de vista operacional, es muy simple y con mucha fortaleza estadística (Ecuación 1).

$$
L_{t e}=L_{t}+142,59(\mathrm{~m})
$$

La segunda hipótesis a verificar implica que los conductores se aproximan más a su velocidad deseada a medida que la longitud de recta es mayor. Esto supone relacionar las longitudes de las rectas, tanto geométrica $\left(L_{t}\right)$ como efectiva $\left(L_{t e}\right)$, con el grado de velocidad de operación deseada (GV85d). En las Figuras 3 y 4 se observan las relaciones entre dichas variables y se verifica la hipótesis planteada, aunque aparece cierta dispersión para longitudes reducidas. Esta es debida a la influencia de otras variables, como los radios de las curvas en los extremos y, por tanto, a sus velocidades de operación. También influye el nivel de la velocidad de operación de referencia del segmento, que es la máxima observada a lo largo del mismo. Esta velocidad de operación se corresponde siempre con rectas largas, estando afectada por variables generalmente asociadas a la sección transversal o al entorno. 


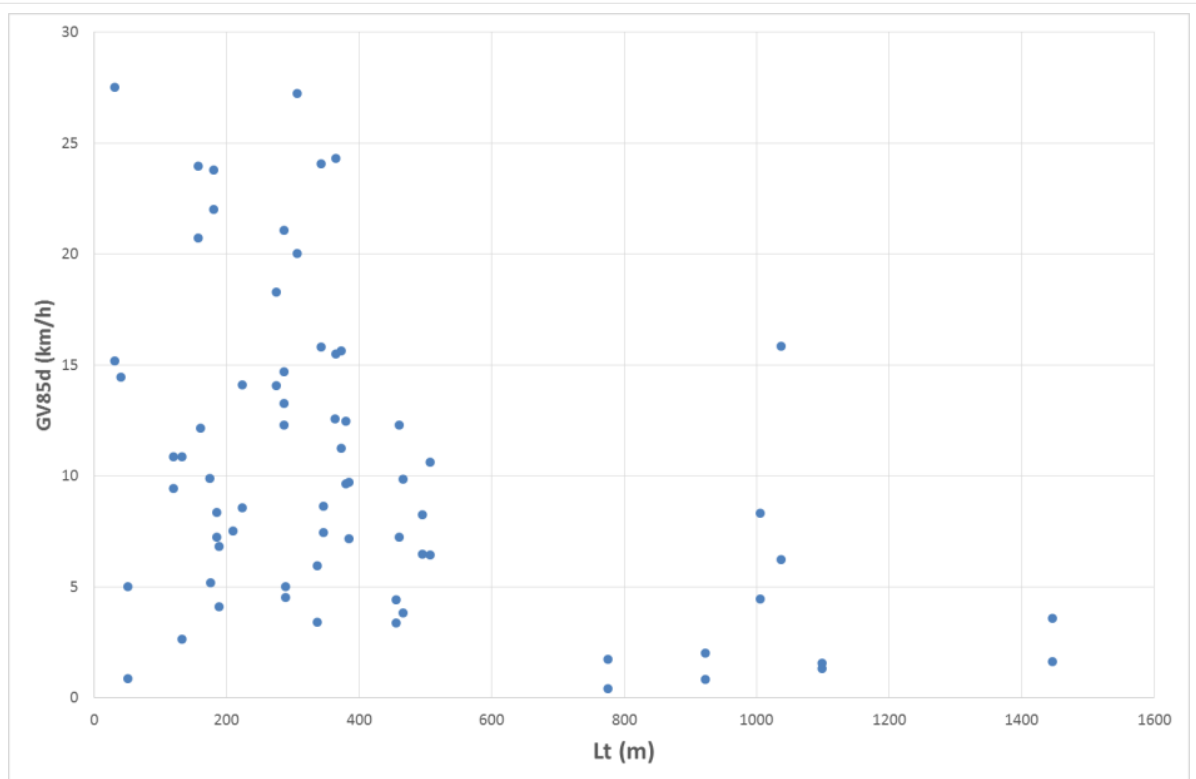

Figura 3 - Relación entre la longitud geométrica de las rectas y el grado de velocidad de operación deseada

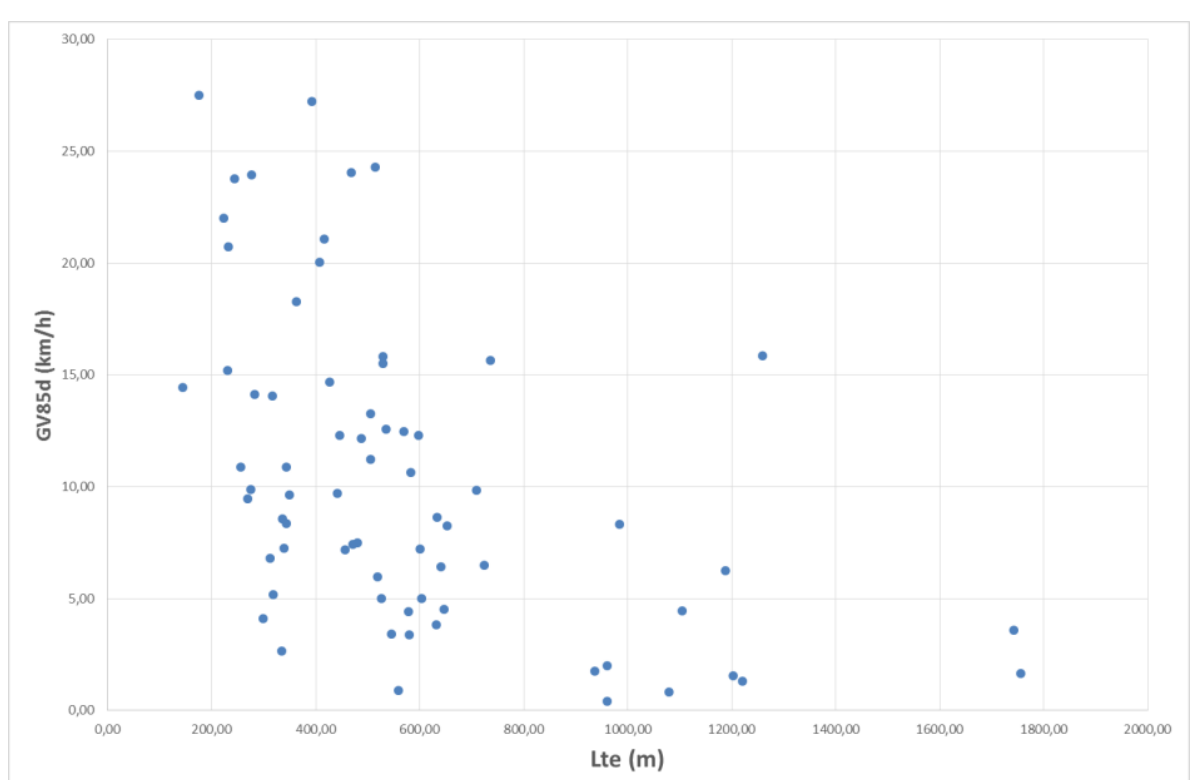

Figura 4 - Relación entre la longitud efectiva de las rectas y el grado de velocidad de operación deseada

La siguiente hipótesis planteada, acorde con la nueva Instrucción de Trazado 3.1 IC (Ministerio de Fomento, 2016), supone que, a menor velocidad de diseño, menor es la longitud mínima de la recta para ser considerada independiente. Para analizar esto, se comparará el grado de velocidad de operación deseada para diferentes rectas para diferentes velocidades de diseño. En la Figura 5 se observa que para el conjunto global de secciones, el grado de velocidad deseada no depende de la velocidad de diseño. Si se agrupan las secciones en tres subgrupos con velocidades de diseño próximas a 50, 60 y $70 \mathrm{~km} / \mathrm{h}$, y se calculan los promedios del grado de velocidad de operación deseada para longitudes de 
rectas menores de 200, 300, 400 y todas ellas, se obtiene la gráfica de la Figura 6. En ella se comprueba que no existe la relación planteada en la hipótesis, por lo que se refuta la misma.

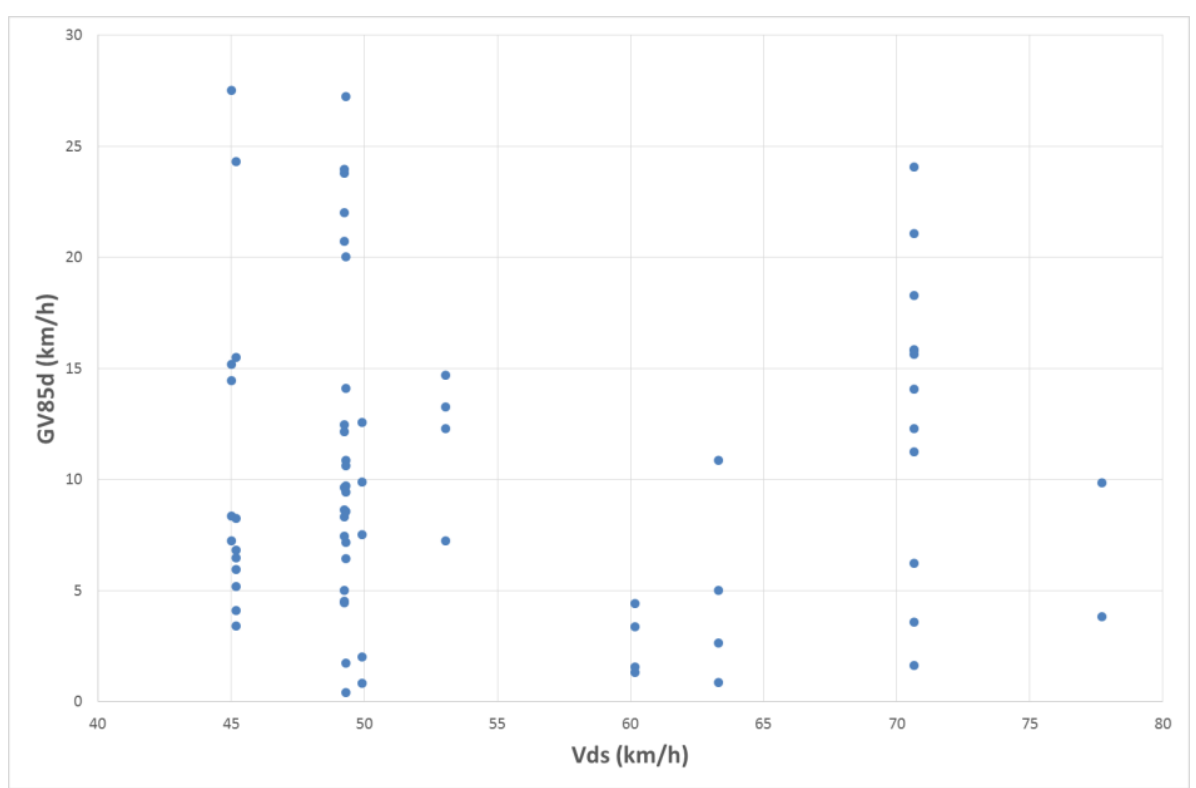

Figura 5 - Relación entre la velocidad de diseño del segmento y el grado de velocidad de operación deseada

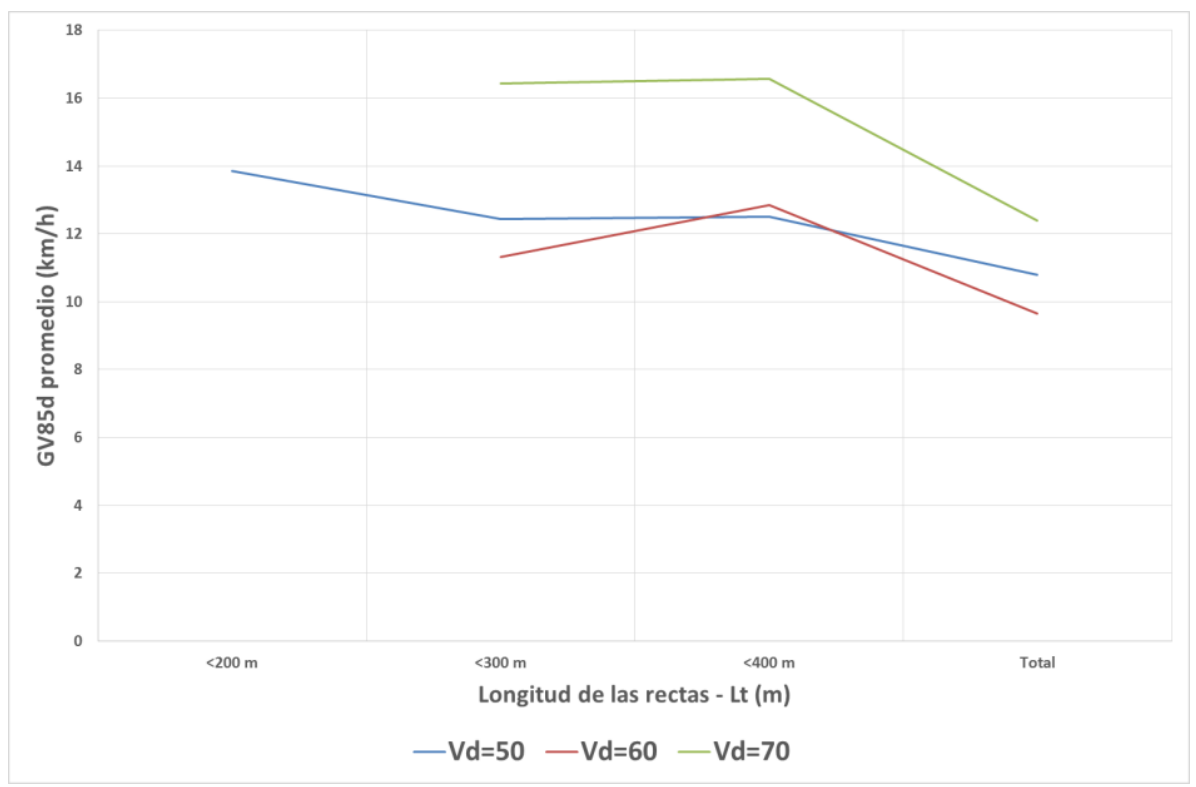

Figura 6 - Relación entre la velocidad de diseño del segmento, las longitudes de las rectas y el promedio del grado de velocidad de operación deseada

La última hipótesis propone que hasta cierta longitud de recta existe condicionamiento operacional entre las curvas de los extremos. Rectas muy cortas implican que no se alcanza la velocidad deseada, aspecto que cambia en rectas largas. Esta conjetura puede ser verificada a través de la influencia que tiene la longitud de la recta en la reducción de la velocidad al final de la recta, es decir, la diferencia entre la velocidad máxima de operación final de la recta (V853js) y la velocidad mínima de operación de la curva 2 (V854js). En la 
Figura 7 se puede observar cómo a mayor longitud de recta hay una tendencia hacia una mayor reducción de velocidad, si bien la dispersión también es elevada. En la figura se han destacado los valores según los umbrales de reducción de velocidad de operación propuestos por Lamm et al. (1999), es decir, 10 y $20 \mathrm{~km} / \mathrm{h}$. Cuanto menor es la reducción de velocidad mayor condicionamiento existe entre las curvas y, por tanto, menos independiente será la recta. Así, se ha calificado la independencia de las rectas en débil, intermedia y fuerte, siguiendo los umbrales de consistencia de Lamm et al. (1999).

Cabe tener en cuenta que la reducción de velocidad dependerá también de la velocidad de operación de la curva final. Es decir, si se trata de una curva cerrada se fomentarán las deceleraciones elevadas, mientras que en curvas suaves la reducción tenderá a ser menor, independientemente de la longitud de la recta. De ahí la dispersión que se puede apreciar en la figura anteriormente referida.

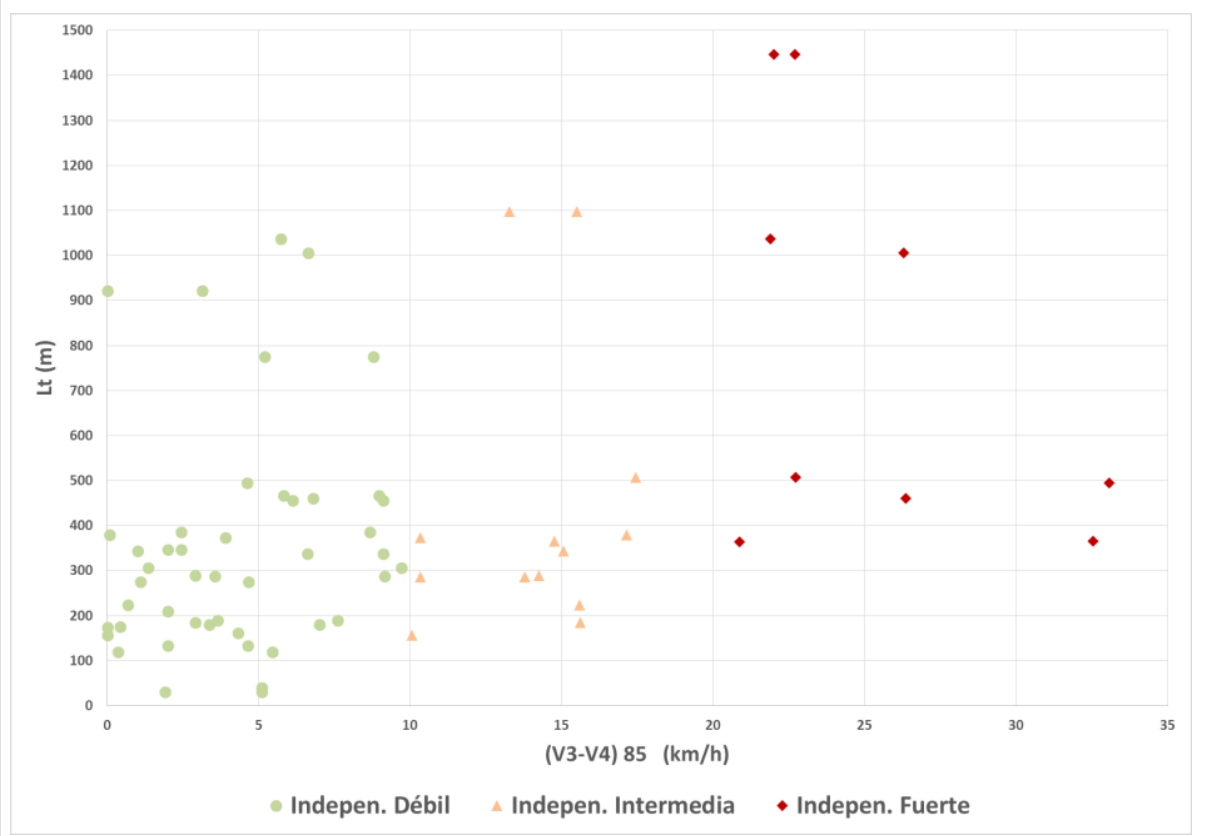

Figura 7 - Relación entre la longitud de la recta y la reducción de velocidad de operación al final de la recta

Otra forma de abordar el contraste de esta hipótesis es a través del análisis de la influencia que tiene la longitud de la recta en la diferencia de velocidad de operación entre la media de las velocidades máximas de operación en la recta (V852js, V853js) y la media de las velocidades de operación de las curvas (V851js, V854js). En la Figura 8 se puede observar cómo a mayor longitud de recta hay una más clara tendencia hacia una mayor variación de la velocidad de operación. En la figura se han destacado los valores según los umbrales de reducción final de velocidad de operación de la figura anterior (10 y $20 \mathrm{~km} / \mathrm{h})$, calificando de la misma forma la independencia de las rectas en débil, intermedia y fuerte. Se vuelve a apreciar que cuanto menor es la variación de velocidad de operación mayor condicionamiento existe entre las curvas y, por tanto, menos independiente es la recta. En 
este caso se ve de forma más evidente el cambio de dispersión entre umbrales, pues se tiene en cuenta tanto la velocidad de partida como la final para cada recta.

Por tanto, a través de los dos análisis realizados se puede verificar la hipótesis formulada, es decir, que cuanta más longitud presenta la recta mayor independencia adquiere al acoger unas variaciones de velocidad de operación mayores.

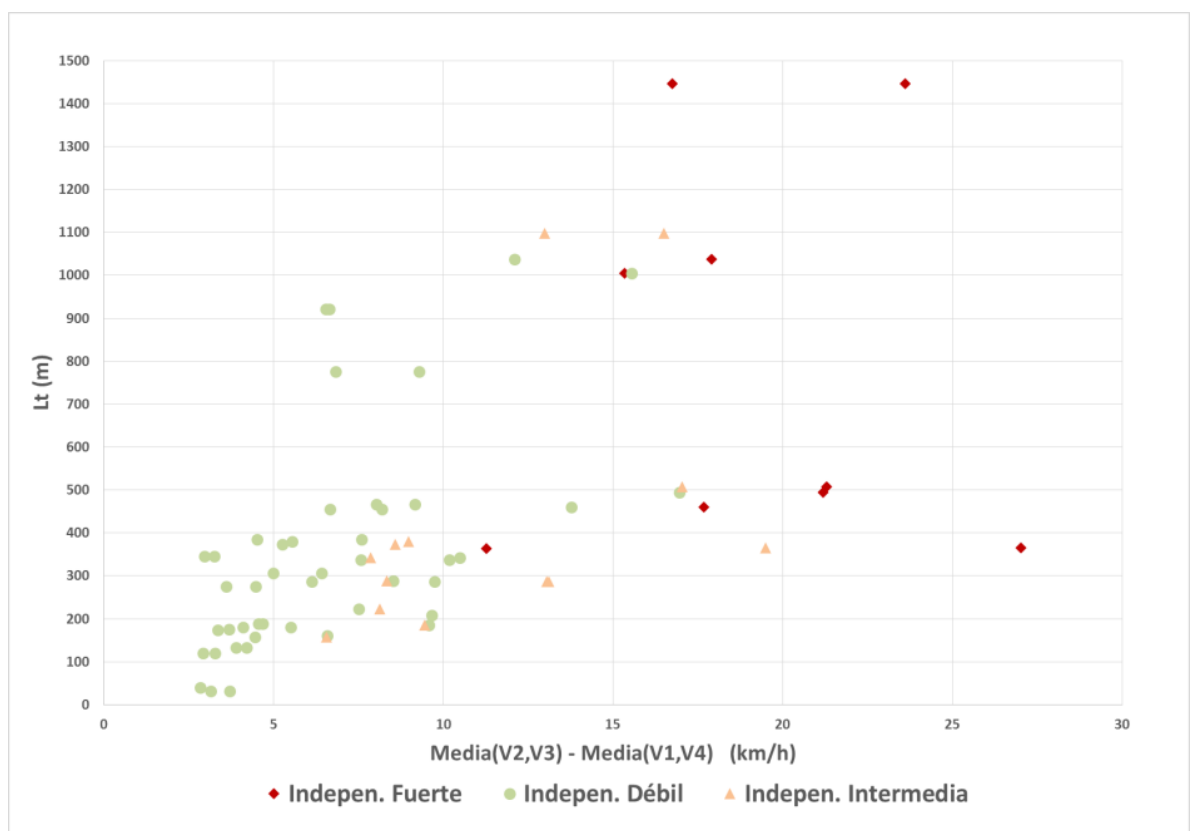

Figura 8 - Relación entre la longitud de la recta y la media de la variación de la velocidad de operación al inicio y final de la recta

\section{DISCUSIÓN}

Una vez contrastadas las hipótesis, se ha realizado una comparación entre los parámetros de las secciones estudiadas y los que la Instrucción 3.1-IC anterior (Ministerio de Fomento, 1999) y la nueva Instrucción 3.1-IC (Ministerio de Fomento, 2016) establecen que deberían tener. Así, de las 70 secciones estudiadas, habría 33 secciones que no cumplirían con la Norma anterior y 43 que no lo harían para la nueva Norma.

En la anterior Norma, el criterio establecido para distinguir rectas independientes de no independientes era muy claro y sencillo, ya que las rectas superiores a 400 metros eran consideradas como independientes sin tener en cuenta otros factores que pueden influir. En cambio, en la nueva Norma (Tabla 2), esta longitud aumenta con la velocidad de diseño. 


\begin{tabular}{|c|c|}
\hline $\begin{array}{c}\text { Velocidad de proyecto }(\mathrm{Vp}) \\
\text { del tramo } \\
(\mathrm{Km} / \mathrm{h})\end{array}$ & $\begin{array}{c}\text { Máxima longitud de una } \\
\text { alineación recta para ser } \\
\text { considerada de longitud limitada } \\
\mathbf{m}\end{array}$ \\
\hline $140,130,120,110$ y 100 & 400 \\
\hline 90 & 300 \\
\hline 80 & 230 \\
\hline 70 & 175 \\
\hline 60 & 85 \\
\hline 50 & 50 \\
\hline 40 & 30 \\
\hline
\end{tabular}

Tabla 2 - Longitud mínima de recta para ser independiente (Instrucción 3.1-IC, 2016)

Esto ha quedado demostrado en el análisis de velocidad en rectas que no se cumple, ya que trazados con radios menores implican menores velocidad de operación en las curvas, y por lo tanto precisarán de mayor longitud en las rectas para ser consideradas independientes. Es decir, la actual Norma no solo no refleja la realidad, sino que el criterio establecido se ha escalado de manera inversa.

Del total de las rectas de las secciones, según la Norma anterior, 22 serían rectas independientes y según la nueva Norma, todas serían rectas independientes excepto cuatro. Esto es muy llamativo, ya que una proporción de rectas independientes del $94 \%$ no parece muy consistente, puesto que según esta misma Norma el diseñador está obligado en dichos casos a aumentar los radios de las curvas adyacentes de una forma muy generalizada a lo largo del trazado. Esto conlleva elevar la velocidad media de operación del segmento, separando aún más la velocidad de operación de la de diseño designada al inicio del proceso.

Para profundizar en el análisis y obtener conclusiones prácticas, partiendo de la Figura 7 se ha calculado el centroide de cada grupo, así como la dispersión media en términos de longitud de recta y reducción última de velocidad. Para identificar mejor los niveles de independencia se han utilizado distintos colores (Figura 9). 


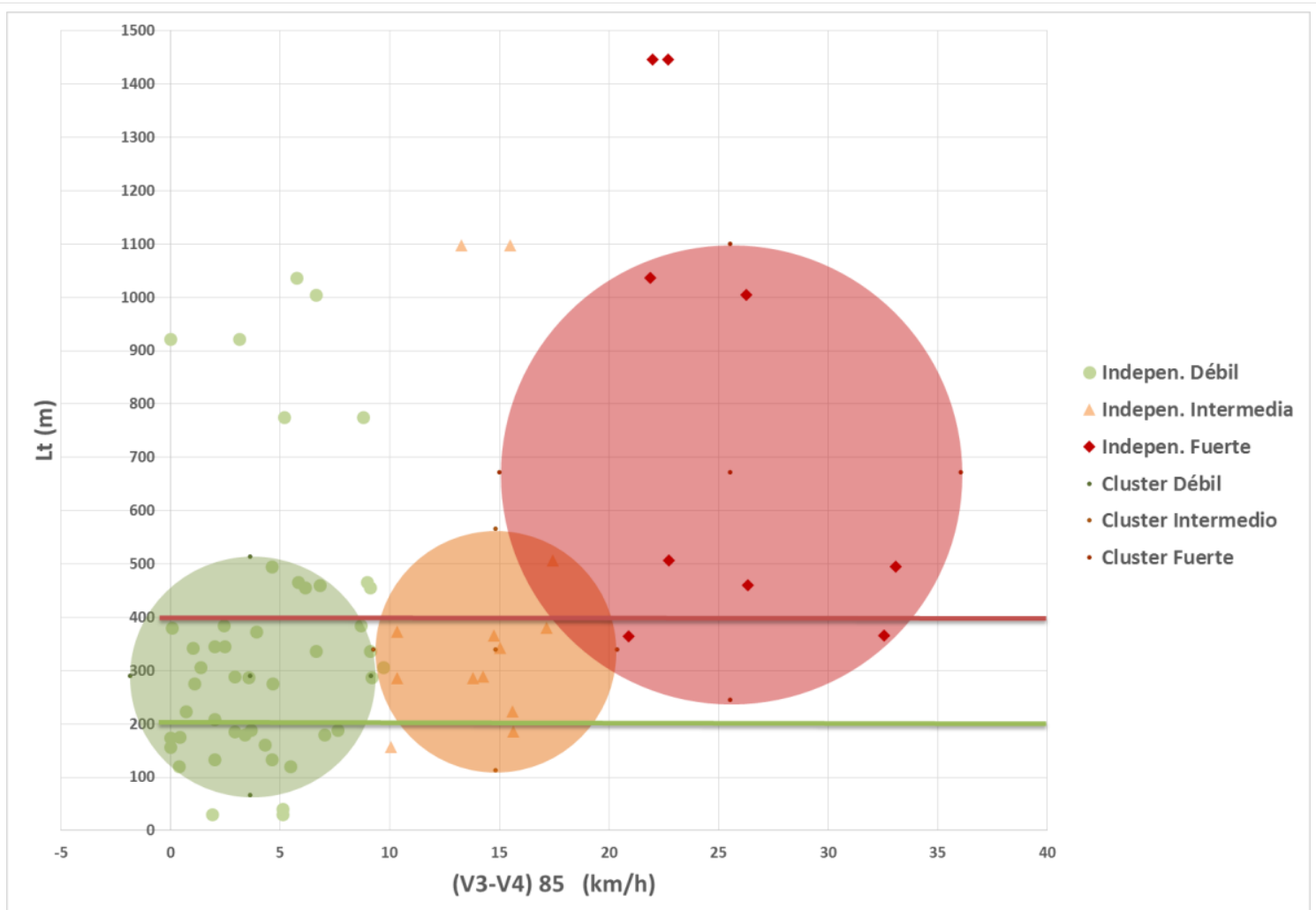

Figura 9 - Niveles de independencia de la recta según la relación entre la longitud de la recta y la reducción de velocidad de operación al final de la recta

Observando el gráfico de la Figura 9, se podrían situar las líneas marcadas en rojo y verde que muestran las fronteras entre los distintos niveles de independencia de las rectas y, por tanto, la mayor o menor holgura de la relación de los radios de entrada y salida. Así, se puede distinguir claramente entre tres grupos de secciones según su longitud: 0-200 metros, 200-400 metros y secciones de longitud superior a 400 metros. Por encima de los $200 \mathrm{~m}$ se encuentran la mayoría de secciones con rectas de independencia intermedia o fuerte, mientras que por encima de los $400 \mathrm{~m}$ se hallan la mayoría de las secciones con rectas de independencia fuerte.

\section{CONCLUSIONES}

La investigación experimental desarrollada ha permitido llegar a diversas conclusiones sobre la influencia de la longitud de las rectas en la consistencia del diseño geométrico. Para ello se han analizado datos existentes, los cuales fueron obtenidos mediante la observación continua de la evolución de una muestra de vehículos ligeros recorriendo nueve tramos de carretera convencional. Se han seleccionado 70 secciones constituidas por una recta entre dos curvas.

La primera conclusión importante es que el comportamiento general de los conductores es iniciar su aceleración antes de que concluya la primera curva y completar su deceleración ya dentro de la segunda curva. Por tanto, se ha encontrado que hay una longitud de recta efectiva 
mayor que la longitud geométrica y el modelo resultante es muy simple, ya que la longitud efectiva parece guardar una relación constante con la longitud geométrica, siendo la primera aproximadamente $145 \mathrm{~m}$ superior a la última, sin que influyan otras variables.

La segunda conclusión se corresponde con la verificación de que a mayor longitud de recta, los conductores se aproximan más a su velocidad deseada. Esta relación presenta cierta dispersión debida a la influencia de otras variables, como las velocidades de operación de las curvas adyacentes. También influye el nivel de la velocidad de operación de referencia del segmento, que es la máxima observada a lo largo del mismo.

La tercera conclusión supone la refutación de la hipótesis que subyace en la propuesta de la nueva Instrucción de Trazado para establecer la máxima longitud de recta para ser considerada de longitud limitada (recta dependiente) en función de la velocidad de diseño (proyecto). En esta investigación ha quedado demostrado que ciertamente hay una relación, pero esta es completamente contraria a la prescrita en la tabla 4.2 de la nueva Instrucción de Trazado.

Al verificar la cuarta hipótesis se concluye que hasta cierta longitud de recta existe condicionamiento operacional entre las curvas de los extremos, por lo que se ha llegado a proponer unos umbrales de longitud de recta que marcan el nivel de independencia de la recta, asociados a las variaciones de velocidad entre la recta y las curvas. Se han propuesto tres grupos de rectas:

- Grupo 1 - rectas muy dependientes: $\mathrm{Lt} \leq 200 \mathrm{~m}$

- Grupo 2 - rectas dependientes: $200<\mathrm{Lt} \leq 400 \mathrm{~m}$

- Grupo 3 - rectas independientes: Lt $>400 \mathrm{~m}$

En el primer nivel se encuentra la zona de relación de radios estricta en la que, según los resultados obtenidos, la proporción entre el radio de entrada y salida no permite apenas variación. Este criterio sólo será válido para valores de longitudes de recta inferiores a 200 metros.

El segundo nivel delimita el rango de longitudes para el que la relación entre radios consecutivos puede tener cierta holgura. Pese a existir una dependencia entre ambos radios, esta no es tan restrictiva como en el caso de rectas inferiores a 200 metros.

Por último, se encuentra el grupo menos restrictivo, conformado por rectas de longitud superior a 400 metros. En este caso, se considera que a partir de esta longitud de recta, el radio de la curva de entrada no tiene influencia sobre el conductor a la hora de afrontar la curva de salida.

Como evolución de esta investigación, se debería fijar una relación de radios para cada 
intervalo de longitud de recta a la vista de los resultados obtenidos, siendo más estricta la proporción de radios para el Grupo 1 que para el Grupo 2. Para el Grupo 3, donde las rectas son independientes, lo que primará será fijar un radio mínimo de las curvas de los extremos y no una proporción máxima entre los radios de las curvas. Después de analizar la normativa anterior y la nueva Norma, parece que la mejor opción para solucionar la problemática es hacer una combinación de las dos. Así, para relaciones de radio estrictas (Grupo 1) se propone utilizar la Norma nueva y para las relaciones de radios intermedias (Grupo 2) la anteriormente vigente, que era menos estricta. Para poder aplicar la Norma que corresponda en cada caso, bastaría regirse por los umbrales propuestos en la presente investigación. Asimismo, el estudio detallado de estos comportamientos podría permitir el desarrollo de modelos de velocidad de operación en rectas de mayor precisión que los existentes en la actualidad.

Con los resultados obtenidos del análisis de la muestra de secciones, se ha llegado a varias conclusiones que contradicen y complementan la nueva Norma. De la continuación de esta investigación, se podrán proponer criterios alternativos específicos para las características de las rectas y sus curvas en los extremos que estén correlacionados con la mejora de la consistencia del diseño geométrico y, por tanto, con el incremento de la seguridad vial.

\section{AGRADECIMIENTOS}

El desarrollo de esta investigación se enmarca dentro del proyecto "CASEFU - Estudio Experimental de la Funcionalidad y Seguridad de las Carreteras Convencionales", con referencia TRA2013-42578-P, subvencionado por el Ministerio de Economía y Competitividad.

Asimismo, la toma de datos continuos de velocidad en la que está basada este proyecto se llevó a cabo dentro del proyecto "REVEL - Una Metodología para la Revisión de los Límites de Velocidad", de referencia PT-2006-031-25 IAPP, gracias a la subvención parcial recibida del Centro de Estudios y Experimentación de Obras Públicas (Ministerio de Fomento).

\section{REFERENCIAS}

CAMACHO-TORREGROSA, F.J.; PÉREZ-ZURIAGA, A.M., CAMPOY-UNGRÍA, J.M.; GARCÍA, A. Y TARKO, A. (2015). Use of Heading Direction for Recreating the Horizontal Alignment of an Existing Road. Computer-Aided Civil and Infrastructure Engineering, vol. 30(4), pp. 282-299.

EASA, S. (2003). Improved Speed-Profile Model for Two-Lane Rural Highways. Canadian Journal of Civil Engineering, vol. 30, pp. 1055-1065.

FITZPATRICK, K.; ELEFTERIADOU, L.; HARWOOD, D.; COLLINS, J.; MCFADDEN, J.; ANDERSON, I.; KRAMMES, R.; IRIZARRY, N.; PARMA, K.; BAUER, K. Y PASSETI, K. (2000). Speed Prediction for Two-Lane Rural Highways. FHWA Report. FITZPATRICK, K. Y COLLINS, J. (2000). Speed-Profile Model for Two-Lane Rural Highways. Transportation Research Record, vol. 1737, pp. 42-49. 
GARCÍA, A.; LLOPIS-CASTELLÓ, D.; CAMACHO-TORREGROSA, F.J. Y PÉREZZURIAGA, A. (2013). New Consistency Index Based on Inertial Operating Speed. Transportation Research Record, vol. 2391, pp. 105-116.

GARCÍA, A.; CAMACHO-TORREGROSA, F.J. Y MARÍN MORALES, J. (2015). Experimental Determination of the Inertial Operating Speed for Consistency and Segmentation Analysis. Transportation Research Board $94^{\text {th }}$ Annual Meeting, Washington D.C., EEUU.

HASSAN, Y. (2004). Highway Design Consistency: Redefining the State of Knowledge and Practice. Transportation Research Record, vol. 1881, pp. 63-71.

LAMM, R.; CHOUEIRI, E. M. Y MAILAENDER, T. (1992). Traffic safety on two continents - a ten year analysis of human and vehicular involvements. Proceedings of the Strategic Highway Research Program (SHRP) and Traffic Safety on Two Continents, pp. $18-20$.

LAMM, R.; PSARIANOS, B. Y MAILAENDER, T. (1999). Highway Design and Traffic Safety Engineering Handbook. Editorial McGraw-Hill.

LAMM, R.; BECK, A.; RUSCHER, T.; MAILAENDER, T.; CAFISO, S. Y LA CAVA, G. (2007). How to Make Two-Lane Rural Roads Safer. Editorial WITPress.

NICHOLSON, A. (1998). Superelevation, Side Friction and Roadway Consistency. Journal of Transportation Engineering. Vol 124, $\mathrm{n}^{\circ}$ 5. pp. 411-418.

OTTESEN, J. Y KRAMMES, R. (2000). Speed-Profile Model for a Design-Consistency Evaluation Procedure in the United States. Transportation Research Record, vol. 1701, pp. 76-85.

PÉREZ-ZURIAGA, A.M.; GARCÍA, A.; CAMACHO-TORREGROSA, F.J. Y D'ATTOMA, P. (2010). Modeling Operating Speed and Deceleration on Two-Lane Rural Roads with Global Positioning System Data. Transportation Research Record, vol. 2171, pp. 11-20.

PÉREZ-ZURIAGA, A.M.; CAMACHO-TORREGROSA, F.J.; CAMPOY-UNGRÍA, J.M. Y GARCÍA, A. (2013). Application of Global Positioning System and Questionnaires Data for the Study of Driver Behavior on Two-Lane Rural Roads. IET Intelligent Transport Systems, vol. 7(2), pp. 182-189.

POLUS, A.; FITZPATRICK, K. Y FAMBRO, D. (2000). Predicting Operating Speeds on Tangent Sections of Two-Lane Rural Highways. Transportation Research Record, vol. 1737, pp. 50-57. 